\title{
Ueber krystallisirtes Cyanhämoglobin.
}

\author{
Von
}

Dr. Rich. v. Zeynek.

(Aus dem Laboratorium für medicinische Chemie in Wien.)

(Der Redaction zugegangen am 10. August 1901.)

Die Frage über das Verhalten von Cyanverbindungen gegen Blutfarbstoff wurde schon vor etwa 35 Jahren von W. Preyer und bald nachher von Hoppe-Seyler untersucht. Obwohl seither mehrere Autoren diese Frage zum Gegenstande ihrer Studien gemacht haben, sind die Resultate derselben keineswegs feststehend, im Gegentheil, beinahe in jeder diesbezüglichen Publication werden andere Anschauungen verfochten. Leider sind aber für die letzteren zwingende Beweise nicht erbracht, sodass es trotz der mannigfachen Bemühungen berechtigt erscheint, dass die Lehrbücher der physiologischen Chemie sich mit den kürzesten Hinweisen darauf begnügen, es seien Verbindungen des Cyans mit Blutfarbstoff beschrieben worden. Daher sei es mir gestattet, vor der Beschreibung meiner Versuche durch einen orientirenden Ueberblick für diese Frage $\mathrm{zu}$ interessiren.

Als erster Beobachter theilte W. Preyer ${ }^{1}$ ) mit, dass das optische Verhalten von Oxyhämoglobinlösungen durch verdünnte wässerige Cyankalium- und Blausäurelösungen in der Kälte nicht verändert wird, dass dagegen beim Erwärmen dieser Mischungen auf Körpertemperatur die Oxyhämoglobinstreifen verschwinden, an ihrer Stelle ein breites Absorptions-

1) Einwirkung des Cyankalium und der Blausäure auf den Blutfarbstoff. Verbessert (nb. auf Grund einer Kritik Hoppe-Seyler's) abgedruckt aus dem Centralblatt f. d. med. Wissenschaften vom 13. April 1867 in W. Preyer, die Blausäure, 1868. 
band erścheint, jenem des sauerstofffreien Hämoglobins ähnlich; nur mehr dem Violett genähert. Von Hämoglobinlösungen unterschied sich diese Lösung auch noch durch ihre mehr gelbrothe Farbe und ihre Resistenz gegen den Sauerstoff der Luft, welcher die Oxyhämoglobinstreifen nicht mehr hervorrufen konnte. Schwefelammoniumzusatz gab auffallende Aenderungen des Spectrums, aber trübe Flüssigkeiten. Eine Lösung von Kohlenoxydhämoglobin veränderte sich mit Blausäure und verdünnten Cyankaliumlösungen, auf $40^{\circ}$ erwärmt, nicht.

In einer weiteren Arbeit beschreibt Preyer ${ }^{1}$ ) eingehend die Spectralerscheinungen seines Blutfarbstoffderivates, er theilt ferner mit, dass es ihm gelungen ist, den aus Oxyhämoglobin vom Hunde durch Blausäureeinwirkung erhaltenen Körper krystallisirt darzustellen. Im trockenen Zustande ist derselbé geruchlos; mit höchst verdünnter Phosphorsäure destillirt, liefert er Blausäure.

In demselben Jahre erschien eine Mittheilung HoppeSeyler's ${ }^{2}$ ) über eine unzweifelhafte Cyanwasserstoffverbindung «mit den Hämoglobinverbindungen in den Lösungen des Meerschweinchen- sowie deș Hundeblutes». Die entstandenen Verbindungen schieden sich nach Zusatz von 1/4 Volumen Alkohol beim Abkühlen unter $0^{0}$ in derselben Weise und in denselben Formen ab, als wäre keine Blausäure zugegen'. Die Krystalle sind jedoch blausäurehaltig, wie die Destillation mit verdünnter Schwefelsäure ergab; ihr Blausäuregehalt (über welchen aber keine Zahlenangaben mitgetheilt sind) veränderte sich beim Umkrystallisiren wie beim Trocknen mit der Luftpumpe über Schwefelsäure nicht. «Es ist diese Verbindung sogar beständiger als die reinen Oxyhämoglobinverbindungen .... Als auffallend hebt Hoppe-Seyler hervor, dass 1. die Lichtabsorption eine deutliche Abweichung von jener der reinen Oxyhämoglobinlösungen nicht zeigte; 2. die Blutkörperchen selbst die Blausäure nicht in chemische

1) Die Ursache der Giftigkeit des Cyankaliums und der Blausäure. Verbessert abgedruckt aus Virchow's Archiv f. pathol. Anat. etc. vom 5. Sept. 1867 in W. Preyer, die Blausäure.

2) Medicinisch-chemische Untersuchnugen. S. 206. 
Verbindung aufzunehmen schienen, denn im Blute eines mit Blausäure getödteten Hundes war schon nach der Isolirung der Blutkörperchen in letzteren kaum eine Spur von Blausäure nachweisbar. Schwefelammon bewirkte die Bildung von reducirtem Hämoglobin. Schliesslich stellt Hoppe die Angaben Preyer's über das spectrale Verhalten seiner Substanz als nicht sicher hin und spricht die Vermuthung aus, dass bei dessen Versuchen in Folge einer Spaltung des Blutfarbstoffs Cyanhämatin gebildet worden sei.

Trotzdem diese Mittheilung Hoppe's den Schluss auf die Existenz einer recht festen Verbindung von Blausäure mit Blutfarbstoff erlaubt, scheint Hoppe-Seyler selbst in den Inhalt dieser Mittheilung Zweifel gesetzt zu haben - möglicher Weise erhielt er bei späteren Versuchen diese cyanhaltige Verbindung nicht - , so dass er in seinem Lehrbuch ${ }^{1}$ ) von einer sehr lockeren Verbindung des Cyanwasserstoffs mit Oxyhämoglobin spricht und als für diese Verbindung charakteristisch hervorhebt, dass sie durch Wasserstoffsuperoxyd in Cyanhämatin und Eiweiss zerlegt werde, während eine $\mathrm{Oxy}$ hämoglobinlösung unverändert bleibe.

Krukenberg') wiederum bestätigt Preyer's Beobachtungen über Bildung und Spectralverhalten des Blausäurehämoglobins und hält Hoppe's Verbindung für nicht einheitlich. Krukenberg's erster Verdacht, ein Zersetzungsprodukt erhalten zu haben oder vielleicht reducirtes Hämoglobin, dessen Spectrum durch den Salzgehalt der Flüssigkeit etwas modificirt wäre, erwies sich ihm als ungerechtfertigt.

Jene Arbeiten, welche sich mit der Veränderung von Blutfarbstoff durch Dicyan ${ }^{3}$ ) beschäftigten, kann ich hier übergehen, da sie einander widersprechen, lediglich als Vorversuche aufzufassen sind und überdies zeigen, dass die Verbindung von Dicyan, über dessen Reinigung auch nichts angegeben ist, mit Blutfarbstoff von störenden Nebenreactionen begleitet war.

1) Physiologische Chemie, 1881, S. 384.

2) Zur Kenntniss der Hämoglobinderivate. Ref. im Jahresber. f. Thierchemie, 1886, S. 111.

3) Laschkewitsch 1868, Lankester 1869, Preyer 1871. 
Hingegen schien eine schöne Beobachtung R. Kobert's geeignet, den Widerspruch in den Resultaten Hoppe-Seyler's und Preyer's aufzuklären. In einer Monographie') hat Kobert beschrieben, dass das Methämoglobin in Bezug auf sein Verhalten gegen Blausäure in bemerkenswerthem Gegensatze stehe zum Oxyhämoglobin wie zum reducirten Hämoglobin. Methämoglobinlösungen werden durch diese schon bei Zimmertemperatur rasch verändert, indem die braunen Lösungen sich roth färben; diese Lösungen zeigen ein vom Spectrum des Hämoglobins wenig verschiedenes Spectrum, nämlich nur einen breiten Streifen im Grünen. Es wird eingehend darauf hingewiesen, welches Interesse dieses bisher unbekannte Blutfarbstoffderivat für den Gerichtsarzt beanspruche. Nach dem Vor- und Schlussworte des Verfassers sollte durch seine Arbeit ein dunkles Gebiet der Toxicologie geklärt werden; das Gleiche bezweckt wohl eine jüngst erschienene Publication desselben Autors. $\left.{ }^{2}\right)$ Kobert nennt vorläufig dieses Veränderungsprodukt Cyanmethämoglobin, ohne Kenntniss seiner elementaren $\mathrm{Zu}$ sammensetzung; er fasst die Blausäurewirkung auf das Methämoglobin als eine Reduction auf und würde das entstehende. Produkt am liebsten Hämoglobin ${ }^{3}$ ) nennen, wenn es nicht beim Schütteln mit Luft unverändert bliebe. Alkalische Methämoglobin]ösungen wurden durch Cyankalium in der gleichen Weise verändert.

Kobert's Cyanmethämoglobin wird als beständig gegen Schwefelammonium beschrieben, ferner gegen Fäulniss beständig bis zum Eintritt stinkender Fäulniss, erwies sich dagegen als fast allen Einflüssen, welche das Methämoglobin in Hämatin oder Hämochromogen umwandeln, in gleicher Weise unter-

1) Ueber Cyanmethämoglobin und den Nachweis der Blausäure, Stuttgart, F. Enke's Verlag, 1891.

2) Beiträge zur Kenntniss der Methämoglobine. Pflüger's Arch., Bd. 82 , S. 603.

3) S. 40 der Monographie: \&Ich glaube nun, dass a uch die Wirkung der CNH auf Methb. nichts anderes ist als eine Reduction, und ich würde das dabei entstehende Produkt am liebsten $\mathrm{Hb}$ nennen, wenn es nur beim Schütteln mit Luft sich nicht ganz anders verhielte als $\mathrm{Hb}, \mathrm{d}$. h. wenn es nur in $\mathrm{O}_{2} \mathrm{Hb}$ übergehen würde.s. 
worfen und dadurch als Hämoglobinderivat charakterisirt. In der zweiten citirten Schrift vertheidigt Kobert erfolgreich die Existenz seines «Cyanmethämoglobins» als Blutfarbstoffderivat, nicht Spaltungsprodukt des Blutfarbstoffs, gegen die Angriffe forensisch-chemischer Autoren, ohne neue Thatsachen bringen zu müssen.

Die Einwirkung von Blausäure auf Methämoglobin, welche eine in der That auffallende und elegante Reaction ist, schien mir eines eingehenden Studiums werth $\mathrm{zu}$ sein. Ich hoffte durch die Untersuchung dieses Reactionsproduktes zur Lösung der Frage über den Unterschied zwischen Methämoglobin und Oxyhämoglobin beizutragen, andererseits schien es bei der geringen Zahl gut charakterisirter Blutfarbstoffderivate sicher von Interesse $\mathrm{zu}$ sein, ein neues darzustellen und $\mathrm{zu}$ studiren. Ich habe seiner Zeit ${ }^{1}$ ) die Vermuthung ausgesprochen, dass die Atomgruppen, welche an Stelle des beim Oxyhämoglobin locker gebundenen Sauerstoffmoleküls sich an den Hämoglobinkern anhängen, beim Methämoglobin zwei Hydroxylgruppen sein mögen. Für diese Vermuthung spricht auch der dem Oxyhämoglobin gegenüber stärker saure Charakter des Methämoglobins. Es lässt sich so die Umänderung des Methämoglobinspectrums beim Zusatze von Alkalien erklären, indem der Wasserstoff dieser Hydroxylgruppen durch Metall ersetzt würde, und in gleicher Weise, stellte ich mir vor, könnte bei Einwirkung von Blausäure eine Ersetzung der Hydroxylgruppen durch Cyangruppen unter Wasserabspaltung eintreten.

Ich habe vorerst eine Reihe orientirender Versuche angestellt, die mich überzeugten, dass thatsächlich die Blausäure, auch in concentrirten wässerigen Lösungen verwendet, keine Hämatin- oder Hämochromogenspaltung von Blutfarbstofflösungen bewirkt, dass dabei auch kein "Säurehämoglobin» ${ }^{2}$ ) gebildet

1) Vergl. Arch. f. Anat. u. Physiol., 1899. Physiol. Abth. S. 460: Neue Beobachtungen und Versuche über das Methämoglobin und seine Bildungsweise. Aus dem physiol.-chem. Institute zu Tübingen.

2) Vergl.E. Harnack, Zeitschr. f. physiol. Chemie, Bd. XXVI, S. 558. 
wiird, dass ferner reine Hämoglobinlösungen wie Kohlenoxydhämoglobinlösungen in ihrem spectralen Verhalten durch Blausäure bei Zimmertemperatur keine. Veränderung im Laufe mehrerer Stunden erleiden. Hämoglobinlösungen konnten in zugeschmolzenen Röhren mit Blausäure sogar stundenlang auf $40^{\circ}$ erwärmt werden, ohne eine Veränderung zu erleiden, und blieben auch nach monatelangem Stehen im vollen Tageslichte unverändert.

Oxyhämoglobinlösungen wurden bei mehrstündigem Erwärmen mit Blausäure auf $40^{\circ}$ in der Weise, wie es Preyer angegeben hat, verändert. Es entstand ein breiter Absorptionsstreifen im Grünen, welcher beim Schütteln der Lösung mit Luft bestehen blieb. Ich habe nun Hämoglobinlösungen, welche mit Blausäure behandelt waren, durch Schütteln mit Luft in Oxyhämoglobin verwandelt und dieses durch Zusatz von 1/4 Volumen Weingeist unter $0^{0}$ krystallisirt erhalten; die gewonnenen Krystalle wurden durch Centrifugiren von der Mutterlauge getrennt, in Wasser von $40^{\circ}$ aufgelöst und aus dieser Lösung nochmals krystallisirt, hierauf nach Entfernung der Mutterlauge unter Zusatz von verdünnter Schwefelsäure destillirt. Das Destillat erwies sich frei von Blausäure. In analoger Weise wurde nachgewiesen, dass reine Oxyhämoglobinlösungen, bei welchen nicht die geschilderte Spectraländerung erfolgt war, und Kohlenoxydlösungen keine Blausäure aufnehmen.

War dagegen in den Oxyhämoglobinlösungen durch die Blausäure, bei tagelangem Stehen derselben mit der Blausäure bei Zimmertemperatur, oder nach stundenlangem Erwärmen auf $40^{\circ}$ schon eine Aenderung des Spectrums eingetreten, so liessen sich Blutfarbstoffkrystalle gewinnen, deren Lösungen bei der Destillation ein blausäurehaltiges Destillat gaben, obwohl die Krystalle selbst nicht den mindesten Geruch nach Cyanwasserstoff hatten. Diese Krystalle waren nicht ebenso leicht $\mathrm{zu}$ erhalten, wie die ursprünglichen Oxyhämoglobinkrystalle; sie erwiesen sich als zerfliesslicher und schon zu ihrer Abscheidung bedurfte es einer tieferen Temperatur.

Wenngleich also eine cyanhaltige Blutfarbstoffverbindung auf diese. Weise zu gewinnen war, so mahnten die unsicheren 
Resultate früherer Autoren und Hoppe-Seyler's Kritik zur Vorsicht, diese Verbindung als einheitlich ohne eingehende Prüfung nicht anzusehen.

Eine glatte und rasche Blausäureanlagerung erfolgt hingegen beim Zusammenbringen von Methämoglobin und Blausäure, dabei tritt, wie ich mich wohl überzeugt habe, keine Gasentwicklung auf; man schien von vornherein berechtigt, nachdem jetzt das Methämoglobin als chemisches Individuum hinreichend charakterisirt ist, das gebildete Produkt als einheitlich anzusprechen. .Eine concentrirte Lösung von Oxyhämoglobinkrystallen, welche bei längerem Stehen im Eiskasten bekanntlich oft, sogar zum grösseren Theile, in Methämoglobin verwandelt sind, gibt auf Blausäurezusatz den von Kobert beschriebenen Farbenumschlag in ausgezeichneter Weise. So ist wohl Hoppe's erstes positives Resultat zu erklären, denn aus dieser methämoglobinreichen Lösung ist nunmehr ein $\mathrm{Ge}$ misch von Oxyhämoglobin mit cyanhaltigem Blutfarbstoff in Krystallen zu erhalten.

Nachdem durch diese Vorversuche eine gewisse W'ahrscheinlichkeit geboten war, eine cyanhaltige Verbindung des Blutfarbstoffes in reinem Zustande gewinnen zu können, wurde alsbald die Darstellung derselben in grösserer Menge versucht.

Als Ausgangsmaterial diente krystallisirtes Oxyhämoglobin vom Pferde, welches sich hier relativ leicht in grosser Menge beschaffen lässt. Eine möglichst concentrirte Lösung der Krystalle wurde mittels einer frisch bereiteten Lösung von rothem Blutlaugensalz, welches durch Umkrystallisiren gereinigt war und dann zum Gebrauche im Dunkeln aufbewahrt wurde, versetzt, bis die vollständige Umwandlung in Methämoglobin erzielt war. Aus dieser eingekühlten Lösung wurde das Methämoglobin durch etwa $20 \%$ kalten Weingeistes in der Weise abgeschieden, wie dies schon wiederholt beschrieben- ist. ${ }^{1}$ ) Die mit der Centrifuge von der Mutterlauge abgetrennten Krystalle wurden mit wenig eiskaltem Wasser gewaschen, in der Regel ein- bis zweimal umkrystallisirt und nun mit einer

1) Vergl. u. A. Arch. f. Anat. u. Physiol., 1899, physiol. Abth. S. 462. 
$1 / 2 \%$ igen wässerigen Blausäurelösung versetzt: Fast momentan löst sich der Krystallbrei auf den Zusatz der Blausậure auf, gleichzeitig schlägt die rehbraune Farbe in eine Rothfärbung um, welche bis auf einen Stich ins Gelbe der Farbe von 0xyhämoglobinlösungen gleichkommt. Das Spectrum dieser Lösung besitzt nur ein breites Absorptionsband im Grünen. Die so erhaltene Lösung wurde mit destillirtem Wasser soweit verdünnt, bis ihr Farbstoffgehalt 25-30\% betrug; und nach dem Einkühlen mit $1 / \$$ ihres Volumens auf $0^{0}$ abgekühlten Alkohols versetzt. Es bedurfte meist einer Temperatur von $-10^{\circ}$, um in 1-2. Tagen eine reichliche Ausscheidung mikroskopischer Krystalle zu erzielen.

Wie beim Methämoglobin vom Pferde erhielt ich auch hier zweierlei Krystallformen; entweder lange Prismen, auf deren einem Ende häufig eine stumpfe Pyramide aufgestellt war. Diese Krystalle waren in der Wärme leicht zerfliesslich, etwa jenen des Oxyhämoglobins vom Rinde vergleichbar. In wenigen Fällen, in welchen die Abscheidung der Krystalle viel langsamer erfolgt war, krystallisirte der Blutfarbstoff in Rhomben aus, welche letzteren gegen die Wärme. wesentlich widerstandsfähiger waren. Einen Grund, weswegen in einem Falle diese oder jene Krystallform auftrat, vermag ich nicht anzugeben; es sei hervorgehoben, dass die Krystalle jeder Darstellung einheitlich aussahen, dass ich ferner in einem Falle bei dem Umkrystallisiren der Prismen das Auftreten der rhombischen Krystalle beobachtet habe.

In jedem Falle wurde durch zwei- bis dreimaliges Umkrystallisiren erreicht, dass nach dem Abtrennen der Mutterlauge mittels der Centrifuge eine nicht nach Blausäure riechende Krystallmasse übrig blieb, welche über Natronkalk, und Schwefelsäure im Vacuum getrocknet harte Krusten gab, die nun zu einem feinem Pulver verrieben und nochmals in gleicher Weise getrocknet wurden.

Auf diese Weise wird ein braunrothes, vom trockenen 0xyhämoglobin nicht unterscheidbares, geruchloses Pulver erhalten, welches in Wasser leicht löslich ist und als interessantesten Unterschied vom ursprünglichen Methämoglobin den zeigt, dass

Hoppe-Seyler's Zeitschrift f. physiol. Chemie. XXXIII. 
Alkalizusatz keine Aenderung des Spectrums hervorruft.

Die gleiche Verbindung konnte aus alkalischen Methämoglobinlösungen durch Zusatz von Alkalicyaniden erhalten werden, nur gelang es ebensowenig, wie es aus irgend einer alkalischen Blutfarbstofflösung bisher gelingen ist, Krystalle von Blutfarbstoff darzustellen. Selbstverständlich muss ein reines, nicht zersetztes Alkalicyanid zur Ausführung dieser Reaction verwendet werden, da sonst begreiflicher Weise eine rasche Zersetzung des Blutfarbstoffes eintritt.

Obwohl die hier beschriebene Darstellung von Methämoglobin ausgeht; möchte ich mir gleich den Vorschlag erlauben, den im Titel dieser Studie schon benützten Namen "Gyanhämoglobin» für den dargestellten Körper an Stelle des provisorisch von Kobert ${ }^{1}$ ) verwendeten Namens Cyanmethämoglobin zu verwenden, und hoffe, im Folgenden die Berechtigung dieses Vorschlages zu erweisen.

Wurde das im Vacuum getrocknete Pulver der Cyanhämoglobinkrystalle im Strome des trockenen Wasserstoffs auf $105-110^{0}$ zur Gewichtsconstanz erwärmt, so erlitten

1. $3,2060 \mathrm{~g}$ Cyanhämoglobin einen Gewichtsverlust von $0,1884 \mathrm{~g}$, entsprechend 5,877\%

2. 11,1713 g Cyanhämoglobin einer anderen Darstellung $0,6546 \mathrm{~g}$, entsprechend $5,860 \%$.

Diese beiden Werthe beziehen sich auf das aus den hygroskopischen Krystallnadeln gewonnene Pulver. Wesentlich andere Werthe erhielt ich bei jenem Pulver, welches aus den rhombischen Krystallen erhalten worden war.

1. Bei $3,1727 \mathrm{~g}$ vacuumtrockenem Krystallpulver wurde ein Gewichtsverlust von 0,3323 g, entsprechend 10,474\%,

2. bei $3,3322 \mathrm{~g}$ ein solcher von $0,3541 \mathrm{~g}$, entsprechend $10,627 \%$, gefunden.

Es ist auffallend, dass gerade die haltbareren Krystalle einen grösseren Gehalt an Krystallwasser aufwiesen, während

1) Monographie S. 14, "vorläufig, ohne dass mir ihre elementare Zusammensetzung bekannt ist, als Cyanmethämoglobin bezeichnen will». 
das Gegentheil zu erwarten gewesen war. Ich dachte, einer Vermuthung folgend, die vor mehreren Jahren Professor v. Hüfner ausgesprochen, an ein etwaiges Vorhandensein von Krystallalkohol, konnte aber bei einer in zweckentsprechender Weise vorgenommenen Destillation im Destillate keinen Alkohol nachweisen.

Der Trockenverlust, welchen die Krystalle erleiden, ist aber nicht ausschliesslich Wasser; es entweicht vielmehr mit demselben eine.kleine Menge von Blausäure, vielleicht auch Cyangas. Um über die Correctur, welche für diese Bestimmungen anzubringen war, eine Vorstellung zu erhalten, wurde in einem Falle an das mit dem Krystallpulver beschickte Gefäss ein Péligot'scher Absorptionsapparat angeschmolzen, in welchen Silbernitratlösung kam. Nach mehrstündigem Erhitzen des Trockengefässes auf $110^{\circ} \mathrm{im}$ Wasserstoffstrome wurde der Silberlösung überschüssiges Ammoniak zugefügt, nach dem Filtriren wurde das durch Salpetersäure ausgefällte Cyansilber bestimmt.

$3,271 \mathrm{~g}$ Krystallpulver lieferten $0,0190 \mathrm{~g}$ Cyansilber, resp. $0,0037 \mathrm{~g}$ Cyan $=0,116 \%$ Cyan oder $0,120 \%$ Cyanwasserstoff.

Nach Abzug etwa dieses Werthes wäre der Trockenverlust als Wasserverlust zu deuten.

Einen zweiten derartigen Versuch habe ich qualitativ durchgeführt, um mit den überdestillirten Wassertropfen die Blausäureproben anzustellen; sowohl die Berlinerblauprobe, wie die Rhodanprobe und die Probe mit Guajactinctur und Kupfersulfat fielen positiv aus.

Es liegt in unseren Krystallen also zweifellos eine Cyanverbindung des Blutfarbstoffes vor. Dass diese Verbindung, wie schon Hoppe-Seyler bei seinem Gemisch von Oxyhämoglobin mit Cyanhämoglobin betont hat, eine recht innige ist, ergibt sich auch daraus, dass das Vacuum selbst bei anhaltendem Erwärmen solcher Lösungen auf $40^{\circ}$ trotz des lebhaften Siedens derselben nicht die mindeste Abspaltung von Cyanwasserstoff erzielt. Unter normalem Luftdruck destillirt, gibt hingegen eine Cyanhämoglobinlösung ein blausäurehaltiges Destillat. In einem Falle wurde die Bestimmung der abdestillirten Säure durchgeführt und dabei ein unbedeutend grösserer Werth er- 
halten, als der oben mitgetheilte, anlässlich der Bestimmung des Krystallwassers gefundene Werth.

Diese beiden Versuche konnten natürlich nur als orientirende Versuche gelten, da ein Theil des angelagerten Cyans, resp. des Cyanwasserstoffs festgehalten sein konnte, vielleicht auch bei der hohen Temperatur verändert worden sein mochte zu einer nicht mehr flüchtigen Verbindung. Um das ganze angelagerte Cyan zu erhalten, habe ich mehrere vergebliche Versuche gemacht, die darauf ausgingen, dasselbe als Berlinerblau oder als Cyansilber nach dem Zerkochen des Cyanhämoglobins mit Laugen zu gewinnen. Schliesslich verwendete ich die folgende Methode.

In die wässerige Lösung des Cyanhämoglobins wurde reichlich fein vertheiltes, gelbes Quecksilberoxyd eingetragen, unter allmählichem Erwärmen mehrere Stunden gerührt. Man konnte annehmen, auf diese Weise das ganze Cyan als Cyanquecksilber in Lösung zu bekommen. Nachdem der ganze Blutfarbstoff gefällt worden war, wurde noch einige Zeit auf dem Wasserbade erwärmt, hierauf filtrirt und mit heissem, destillirtem Wasser das gebildete Quecksilbercyanid ausgewaschen. Diese Lösung wurde auf dem Wasserbade eingeengt, in einer verschlossenen Flasche mit Schwefelwasserstoffgas zerlegt, hierauf wurde zur Entfernung des überschüssigen Schwefelwasserstoffs eine salzsäurefreie Lösung von Cadmiumnitrat zugefügt und nach Ausfällen des Schwefelwasserstoffs wurde in eine verdünnte Silbernitratlösung filtrirt, der eine geringe Menge von Salpetersäure zugesetzt war. Das so gefällte Cyansilber wurde auf einem Ludwig'schen Glaswollfilter gesammelt und gewogen oder nach Rose im Porzellantiegel durch Wasserstoff in metallisches Silber verwandelt und als solches gewogen.

Die ersten Versuche, die ich nach dieser Methode anstellte, zeigten nicht unbeträchtliche Differenzen in ihren Resultaten. Ich wurde durch sie erst aufmerksam, wie schwierig es ist, die letzten Spuren des bei der Methämoglobindarstellung verwendeten Blutlaugensalzes durch Umkrystallisiren und folgende anhaltende Dialyse zu entfernen. Ich habe daher bei den 
weiteren Versuchen stets eine Kontrollprobe mit Wasser gekocht, das Filtrat davon eingeengt und mit Quecksilberoxyd in derselben Weise behandelt, wie es oben beschrieben ist; der Cyangehalt, welcher dem noch an den Krystallen haftenden Blutlaugensalz entsprach, wurde durch die Wägung des. schliesslich erhaltenen Cyansilbers ermittelt und von dem Gesammtwerthe abgezogen. Wiederholt überzeugte ich mich, dass meine Proben von Cyanhämoglobin, welche zu den Bestimmungen verwendet wurden, chlorfrei waren.

Auf diese Weise gelangte ich zu den folgenden Resultaten:

1. $200 \mathrm{ccm}$. einer Lösung, welche $6,022 \mathrm{~g}$ trockenes Cyanhämoglobin (prismatische Krystalle) enthielt (ihre Concentration wurde durch Abdampfen einer Probe im Wasserstoffstrome und Wägen des bei $110^{\circ}$ getrockneten Rückstandes bestimmt) gaben $0,0525 \mathrm{~g}$ Cyansilber $=0,0102 \mathrm{~g}$ Cyan oder $\mathbf{0 , 1 6 9} \%$. Die Kontrollprobe gab nur eine geringe Opalescenz, herrührend von Cyansilber, eine Bestimmung desselben wurde nicht versucht.

2. $17,50 \mathrm{~g}$ trockenes Cyanhämoglobin (prismatische Krystalle) gab 0,1533 g Cyansilber, entsprechend 0,0298 g Cyan oder $0,170 \%$. Auch hier war die Kontrollprobe beinahe negativ.

3. Verwendet wurde eine Lösung der rhombischen Krystalle, die 16,263\% trockenes Cyanhämoglobin enthielt. Von dieser Lösung gaben $88,02 \mathrm{~g}$, entsprechend $14,315 \mathrm{~g}$ Cyanhämoglobin, $0,1165 \mathrm{~g}$ metallisches Silber, welches aus dem Cyansilber durch Glühen im Wasserstoffstrome nach Rose erhalten wurde, entsprechend $0,02811 \mathrm{~g}$ oder $0,196 \%$ Cyan. Zur Kontrollprobe wurden 80,96 g derselben Lösung $(13,167 \mathrm{~g}$ Cyanhämoglobin) verwendet; diese lieferten $0,0218 \mathrm{~g}$ metallisches Silber, entsprechend $0,0053 \mathrm{~g}$ oder $0,040 \%$ Cyan. Dem reinen Farbstoff kommen demnach 0,156\% Cyan zu.

4. Verwendet $170 \mathrm{ccm}$. einer Cyanhämoglobinlösung, deren Concentration spectrophotometrisch mittels der im Folgenden mitgetheilten optischen Constanten bestimmt wurde. Die photometrische Untersuchung der auf das Hundertfache verdünnten Lösung, in der bekannten Weise durchgeführt, ergab für ein Hüfner'sches Spectrophotometer 


$$
\begin{array}{lll}
\varphi=59,16 & \epsilon=0,58038 & \epsilon^{\prime} \\
\varphi^{\prime}=63,58 & \epsilon^{\prime}=0,70338 & 1,212
\end{array}
$$

Aus $\epsilon$ berechnet sich die Concentration der ursprünglichen Lösung zu 10,62, aus $\epsilon^{\prime} \mathrm{zu} 10,66 \%$, im Mittel 10,64. Die $170 \mathrm{ccm}$. der Lösung enthielten also 18,09 g Cyanhämoglobin. Gewonnen wurde $0,1085 \mathrm{~g}$ metallisches Silber, entsprechend 0,0261 g Cyan oder 0,144\% Cyan. Eine grössere Probe der gleichen Lösung wurde mit Wasser gekocht und filtrirt. Das Filtrat gab auf Zusatz von Eisenchlorid keine Grünfärbung.

5. 3,271 $\mathrm{g}$ wasserfreies Cyanhämoglobin aus vacuumtrockenen Krystallen (Prismen) gaben 0,0237 g Cyansilber, entsprechend 0,0046 g Cyan oder 0,149\%. Die Kontrollprobe fiel negativ aus.

6. Da die Einwirkung von Cyanwasserstoff auf Methämoglobinlösungen rasch erfolgt und bei Verwendung neutraler (nicht alkalischer) Methämoglobinlösungen der Farbenumschlag der Lösung ein sehr auffälliger ist, überdies schon recht geringe Mengen der überschüssig zugesetzten Blausäure sich dem Geruche verrathen, so habe ich zu einer Methämoglobinlösung, deren Methämoglobingehalt ich kannte, eine verdünnte Blausäure von bestimmtem Gehalte zugefügt, bis die braune Farbe der Lösung in ein reines Roth sich verwandelt hatte und bis eben ein leiser Geruch nach Blausäure bestehen blieb. So. wurden für $200 \mathrm{ccm}$. einer 4,265\% igen Methämoglobinlösung $5,22 \mathrm{ccm}$. Zehntelnormalcyanwasserstofflösung $(4,6 \mathrm{ccm}$. einer $1,134 \times 1 / 10 \mathrm{~N}$-Lösung) verbraucht, entsprechend einer Aufnahme von 0,$1590 ; 0$ Cyan.

Im Mittel dieser Versuche wurde also ein Cyangehalt von $\mathbf{0 , 1 5 8} \%$ für das Cyanhämoglobin gefunden.

Das Hämoglobinmolekül hat nach v. Hüfner's maassgebenden Bestimmungen ein Molekulargewicht von 16669; die mitgetheilten Cyanbestimmungen ergeben, dass von einem Methämoglobinmolekül nur ein einziges Molekül Cyanwasserstoff resp. ein Cyanradical (- CN) aufgenommen wird. Es berechnet sich die Cyanaufnahme zu 0,156\% Cyan gegen 0,158\% $\%$ gefundenes Cyan. Es ist nicht zu übersehen, dass die schöne Uebereinstimmung nicht in der Genauigkeit der verwendeten Methode liegt. 
Wenngleich unser Blutfarbstoffderivat hiermit als einheitlicher Körper, nämlich mit Rücksicht auf seine Krystallisirbarkeit, wie auf den constanten Cyangehalt gelten darf, so erschien es doch räthlich, zur weiteren Charakterisirung gleich die genaue optische Untersuchung durchzuführen, in Anbetracht der Wichtigkeit der photometrischen Untersuchungsmethode gerade für die Blutfarbstoffe.

Es wurde schon bemerkt, dass die hellrothe Lösung des Cyanhämoglobins für das unbewaffnete Auge einer $0 x y-$ hämoglobinlösung recht ähnlich sieht und sich von letzterer nur durch einen Schimmer ins Gelbe unterscheidet. Werden entsprechend verdünnte Lösungen durch ein Spectroskop betrachtet, so ist neben einer starken Verdunkelung des blauen Spectraltheiles nur ein im Grünen gelegener, breiter Absorptionsstreifen zu sehen, jenem des reducirten Hämoglobins nicht unähnlich, aber mehr gegen das stärker lichtbrechende Ende des Spectrums zu gelegen als der letztere. Die dunkelste Stelle dieses Streifens liegt nicht in der Mitte des Streifens, sondern dem violetten Ende des Spectrums näher. Anhaltendes Schütteln mit Sauerstoff ändert das Aussehen und die Lage dieses Streifens nicht im Mindesten.

Der auffälligste Unterschied gegen das Methämoglobin ist aber, dass das Spectrum des Cyanhämoglobins keine augenfällige Differenz zeigt, ob die neutrale oder durch überschüssigen Cyanwasserstoff saure Lösung oder eine Lösung nach Zusatz von so viel Alkali untersucht wird, dass durch letzteres noch keine Spaltung des Hämoglobincomplexes statthat.

Zur Charakterisirung des Präparates als eines einheitlichen Körpers kann diese optische Untersuchung nicht genügen; dagegen bewahrt in weitaus höherem Maasse vor Irrthümern die Untersuchung, wie die Lichtintensitäten im Spectrum vertheilt sind.

Eine genaue graphische Darstellung der Lichtvertheilung im Spectrum des Cyanhämoglobins behalte ich mir für eine besondere Abhandlung vor; hier soll das Untersuchungsergebniss für nur zwei Spectralgegenden mitgetheilt werden, für welche die Absorptionsverhältnisse des Hämoglobins, des Oxyhämoglobins, des Kohlenoxydhämoglobins und des Methämoglobins 
in alkalischer Lösung bestimmt worden sind. Diese Spectralregionen sind:

1. Das Intervall zwischen den Wellenlängen $554 \mu \mu$ und $565 \mu \mu$, und

2. das Intervall zwischen $531,5 \mu \mu$ und $542,5 \mu \mu$.

Die Beobachtungen geschahen mittels eines Hüfner'schen Spectrophotometers und in der Weise, wie v. Hüfner und seine Schüler sie durchführen; ${ }^{1}$ ) als Lichtquelle diente ein Auer'scher Gasbrenner, die Weite des Collimatorspaltes betrug $1 / 40 \mathrm{~mm}$. Die zur Photometrie nöthige Verdünnung der Lösungen geschah stets mit einer $0,1 \%$ igen Sodalösung. In der folgenden Tabelle bedeutet $c$ die Concentration, $\epsilon_{\text {cy }}$ und $\epsilon_{\text {cy }}$ die in den oben bezeichneten Spectralregionen gefundenen Extinctionscoefficienten, $A_{c y}$ und $A^{\prime}{ }_{c y}$ die entsprechenden Absorptionsverhältnisse.

\begin{tabular}{|c|c|c|c|c|c|c|}
\hline c & $\epsilon_{\mathrm{cy}}$ & $\epsilon_{\text {cy }}^{\prime}$ & $\frac{\epsilon^{\prime} \mathrm{cy}}{\epsilon_{\mathrm{cy}}}$ & $A_{c y}$ & $A^{\prime}$ cy & Bemerkungen \\
\hline \multirow{3}{*}{0,001382} & 0,75366 & 0,91951 & 1,220 & 0,001834 & 0,001503 & \multirow{5}{*}{$\begin{array}{c}\text { Lösung } \\
\text { nadelförmiger } \\
\text { Krystalle }\end{array}$} \\
\hline & 0,77460 & 0,93650 & 1,209 & 0,001784 & 0,001476 & \\
\hline & 0,76822 & 0,91951 & 1,197 & 0,001799 & 0,001503 & \\
\hline \multirow{2}{*}{0,001167} & 0,64184 & 0,77560 & 1,208 & 0,001818 & 0,001505 & \\
\hline & 0,64492 & 0,77392 & 1,200 & 0,001810 & 0,001508 & \\
\hline 0,001394 & 0,74098 & 0,89968 & 1,214 & 0,001881 & 0,001549 & \multirow{4}{*}{$\begin{array}{l}\text { Bestimmangen des Trocken- } \\
\text { rückstandes ron Lösungen } \\
\text { feuchter Krystallknchen }\end{array}$} \\
\hline 0,001666 & 0,91054 & 1,10310 & 1,211 & 0,001830 & 0,001510 & \\
\hline 0,001214 & 0,68058 & 0,82558 & 1,213 & 0,001784 & 0,001471 & \\
\hline 0,001660 & 0,90048 & 1,07062 & 1,189 & 0,001843 & 0,001550 & \\
\hline 0,000580 & 0,31504 & 0,38404 & 1,219 & 0,001841 & 0,001510 & \multirow{3}{*}{$\begin{array}{c}\text { Lösung } \\
\text { rhombischer } \\
\text { Krystalle }\end{array}$} \\
\hline \multirow{2}{*}{0,000934} & 0,50330 & 0,59866 & 1,189 & 0,0018556 & 0,001560 & \\
\hline & 0,50152 & 0,60 อั22 & 1,207 & 0,001864 & 0,001543 & \\
\hline \multirow{7}{*}{$\begin{array}{c}\text { Concen- } \\
\text { tration } \\
\text { nicht } \\
\text { be- } \\
\text { stimmt }\end{array}$} & 0,90008 & 1,03770 & 1,208 & \multirow{6}{*}{\multicolumn{2}{|c|}{$\overline{0,001829} 0 \overline{0,001516}$}} & \multirow{5}{*}{$=$ Nlittel aus 12 Bestimmungel } \\
\hline & 0,62750 & 0,74746 & 1,191 & & & \\
\hline & 0,67352 & 0,81056 & 1,203 & & & \\
\hline & 0,94832 & 1,13912 & 1,201 & & & \\
\hline & 0,78138 & 0,94280 & 1,207 & & & \\
\hline & & & & \multirow{2}{*}{\multicolumn{3}{|c|}{$=$ Mittel aus 18 Versuchen. }} \\
\hline & & & & & & \\
\hline
\end{tabular}

1) Vergl. Arch. f. Anat. u. Physiol., physiol. Abth., 1894, S. 135 u. ff. 
In der vorstehenden Tabelle weichen weder die mitgetheilten Absorptionsverhältnisse wesentlich von einander ab, noch zeigen die Quotienten der Extinctionscoefficienten $\frac{\epsilon^{\prime}}{\epsilon}$, die das Verhältniss bedeuten, in welchem die Lichtauslöschungen in den beiden gewählten Spectralregionen zu einander stehen, grössere Verschiedenheiten, als sie bei der Untersuchung anderer Blutfarbstoffe gefunden wurden.

Es sei noch bemerkt, dass bei den einzelnen Darstellungen ein wechselnder Ueberschuss von Blausäure verwendet wurde, dass also ebenso, wie der Ueberschuss von Ferricyankalium bei der Bildung von Methämoglobin, hier die überschüssig zugesetzte Blausäure wenigstens innerhalb gewisser Grenzen in ihrer Wirkung bei der Bildung eines einzigen Körpers Halt macht.

Die mitgetheilten Constanten ermöglichen die spectrophotometrische Bestimmung, sowohl des Cyanhämoglobins allein, als auch neben anderen, optisch charakterisirten Blutfarbstoffen. Ein Vergleich dieser Constanten mit jenen des alkalischen Methämoglobins zeigt jedoch, dass photometrische Bestimmungen dieser zwei Blutfarbstoffe neben einander keiner grossen Genauigkeit fähig sind, falls die Untersuchung in den oben gewählten Spectralregionen durchgeführt wird. ${ }^{1}$ )

Hätte Hoppe-Seyler seine «Cyanwasserstoffhämoglobinverbindung " ${ }^{2}$ mittels des Spectrophotometers prüfen können, so wäre es ihm sicher ein Leichtes gewesen, den Unterschied gegenüber dem Oxyhämoglobin zu constatiren.

Die Einheitlichkeit des oben beschriebenen Blutfarbstoffderivates wurde in ungeahnter Weise durch eine Mittheilung, welche J. Bock vor mehreren Jahren über Photomethämoglobin ${ }^{3}$ ) gemacht hat, bestätigt. Bock's schöne Entdeckung, dass Methämoglobinlösungen am Lichte Veränderungen ihrer Farbe

1) Vergl. Arch. f. Anat. u. Physiol., physiol. Abth., 1899, S. 464 und Hüfner, Tabelle ebenda, 1900, S. 46.

2) a. a. 0., S. 207.

3) Skandin. Arch. f. Physiolgie, Bd. 6, S. 299. 
und ihrer Spectralerscheinungen erleiden und dass der dabei entstehende Körper krystallisirt erhalten werden kann, hat lebhaftes Interesse wachgerufen, umsomehr, als dieses Blutfarbstoffderivat das einzige gegen Lichtwirkung nicht beständige zu sein schien. Es gelang Bock, diesen Photomethämoglobin genannten Körper krystallisirt darzustellen und ihn als chemisches Individuum zu charakterisiren.

Bei meinen Versuchen über Photomethämoglobin fiel mir die Aehnlichkeit seines Spectrums mit dem des Cyanhämoglobins auf. Ich beobachtete, dass ersteres sich aus Methämoglobinlösungen nur dann bildet, wenn in der Lösung Ferricyankalium zugesetzt ist. Dass die Entfernung der letzten Spuren von Blutlaugensalz aus einer Lösung von Methämoglobin durchaus nicht leicht ist, wurde bereits mitgetheilt. Man kann bemerken, wie die Lichtwirkung auf das Methämoglobin mit der allmählichen Entfernung des Ferricyankaliums langsamer eintritt, schliesslich vollständig verschwindet.

Ebensowenig wie das Spectrum reiner, blutlaugensalzfreier Methämoglobinlösungen ändert sich bei der Insolation jenes von Lösungen, welche aus mittels Natriumnitrit hergestellten Methämoglobinkrystallen dargestellt worden sind oder von solchen Lösungen, welche aus «spontan» gebildetem Methämoglobin, wie es etwa bei längerem Aufbewahren feuchter Oxyhämoglobinkrystalle entsteht - allerdings selten ganz frei von Oxyhämoglobin - zu erhalten sind.

Setzt man letzteren zwei Lösungen etwas Ferricyankalium zu, so tritt nach kurzdauernder Einwirkung des Sonnenlichtes die charakteristische Umwandlung von Farbe und Spectrum dieser Lösungen ein.

Es gelang mir auch, dieses Photomethämoglobin in mikroskopischen, nadelförmigen Krystallen zu gewinnen. Dieselben wurden durch sorgsame Dialyse vom anhaftenden Blutlaugensalze befreit und erwiesen sich als identisch mit dem beschriebenen Cyanhämoglobin.

6,58 g, durch Wägung des Trockenrückstandes einer gut dialysirten Lösung von Photomethämoglobinkrystallen bestimmt, gaben nach dem Kochen mit Quecksilberoxyd und schliess- 
lichem Ausfällen der erhaltenen Blausäurelösung mit Silbernitratlösung bei dem Glühen des gewonnenen Cyansilbers nach Rose 0,0410 g metallisches Silber, entsprechend 0,0097 g Cyan oder $0,147 \%$ Cyan.

Eine Lösung von Photomethämoglobin, mit kohlensaurem Calcium in reichlicher Menge versetzt und hierauf destillirt, gibt ein blausäurehaltiges Destillat.

Bei der photometrischen Untersuchung des Photomethämoglobins fand ich im Mittel aus drei Fällen den Quotienten $\frac{\epsilon_{\mathrm{cy}}^{\prime}}{\epsilon_{\mathrm{cy}}}=1,201$, ferner $A_{\mathrm{cy}}=0,001826$ und $\left.A^{\prime}{ }_{\mathrm{cy}}=0,001520 ;{ }^{1}\right)$ woraus hervorgeht, dass das Photomethämoglobin vom Pferde mit dem Cyanhämoglobin vom Pferde optisch übereinstimmt.

Nach diesen Erfahrungen schien die Ursache, welche die Veränderung des Methämoglobins in der Sonne bewirkt, leicht auffindbar zu sein; man musste erwarten, dass sie in einer durch das Licht hervorgerufenen Blausäureabspaltung aus dem Ferricyankalium liege. Letztere ist auch nachweisbar.

Reine, frisch bereitete Lösungen von gut umkrystallisirtem Ferricyankalium wurden etwa $1 / 2$ Stunde dem Sonnenlichte ausgesetzt, hierauf mit Weinsäure angesäuert und nach Zusatz eines Ueberschusses von Calciumcarbonat destillirt. Im Destillate liess sich Blausäure sicher nachweisen. Ich habe diesen Versuch mehrmals wiederholt und zur Kontrolle die nicht belichtete, frisch bereitete Lösung des Ferricyankaliums mit Weinsäure und hernach mit viel kohlensaurem Kalk versetzt und destillirt; bei dieser Kontrollprobe erwies sich das Destillat frei von Cyanwasserstoff.

Es war übrigens bei den Versuchen, in welchen durch Lichtwirkung grössere Mengen von Cyanhämoglobin dargestellt wurden, mir aufgefallen, dass die Lösungen nach mehreren Tagen einen deutlichen Geruch nach Blausäure angenommen hatten.

Nach diesen Erfahrungen macht die Blausäure eine Ausnahme von den übrigen Säuren, deren Wirkung auf den Blutfarb-

1) Aus Bock's Curve für Photomethämoglobin habe ich einen etwas grösseren Quotienten berechnet. 
stoff dahin geht, dass die Bindung zwischen dem farbgebenden Complexe desselben und dem Globin gelockert wird; wahrscheinlich theilt sie ihre Indifferenz noch mit der Kohlensäure. ${ }^{1}$ ) Auf das (reducirte) Hämoglobin selbst konnte ich überhaupt keine Blausäureeinwirkung erzielen: ${ }^{2}$ ) hingegen blieb das Oxyhämoglobin nur in der Kälte unverändert, bei der Temperatur des Brutofens veränderte es sich, wenn nur geringe Blausäuremengen zugesetzt waren, im Laufe einiger Stunden: die Blutfarbstofflösung behielt zwar ihre schöne, rothe Farbe, nimmt aber einen deutlichen gelben Schimmer an, wie schon Preyer ${ }^{3}$ ), der Entdecker dieser Umwandlung, beschrieben hat, und zeigt das Spectrum des Ciyanhämoglobins. Dabei war keine Gasentwicklung in der Blutfarbstofflösung zu beobachten.

Ursprünglich hatte Preyer den analogen Versuch mit Verwendung von Cyankalium statt der Blausäure ausgeführt und die Kritik Hoppe-Seyler's herausgefordert, dass durch die Alkaliwirkung eine Zerstörung des Blutfarbstoffs bewirkt werde.

Verwendet man eine etwa $1 / 2 \%$ ige Blausäure, so tritt jedenfalls keine Hämatinspaltung ein und es gelang relativ leicht, nach dem Abkühlen der Lösungen auf folgenden Weingeistzusatz Krystalle (und zwar immer mikroskopische Nadeln) $\mathrm{zu}$ gewinnen. Preyer berichtet von Trübungen, welche vorher durch Filtriren beseitigt werden mussten; ich habe dabei nie solche beobachtet. Es liegt nicht der mindeste Grund vor, an eine weitergehende Zersetzung des Blutfarbstoffs durch den Cyanwasserstoff zu denken.

Es ist mir nicht gelungen, einen Unterschied zwischen den auf diese Weise erhaltenen Krystallen und der Verbindung, S. 133.

1) Vergl. Hüfner, Arch. f. Anat. u. Physiol. 1894, physiol. Abth.

2) Es sei wiederum betont, dass man sich bei solchen Versuchen von der völligen Abwesenheit des locker gebundenen Sauerstoffs überzeugen muss, da die Anwesenheit des sogenannten Reductionsbandes im Spectrum keineswegs die Gegenwart von Oxyhämoglobin ausschliesst.

3) Die Blutkrystalle 1871. S. 153-155. 
welche aus Methämoglobin mittels Blausäure erhalten worden ist, $\mathrm{zu}$ finden. Eine Cyanbestimmung, durchgeführt mit einer Lösung der feuchten Krystalle, ergab den erwarteten Cyanwerth, auch die photometrischen Versuche gaben die gewünschte Uebereinstimmung. Da auf diese Methode das Cyanhämoglobin am leichtesten rein darstellbar ist, will ich das so erhaltene Produkt als Ausgangsmaterial für weitere Versuche verwenden und theile beiläufig diese Bestätigung der Preyer'schen Versuche, insoweit sie Blausäure und nicht Alkalicyanide betreffen, mit.

Nachdem die beiden Sauerstoffhämoglobine, das Oxyhämoglobin und das Methämoglobin, sich fähig erwiesen haben, nur eine Cyangruppe auf ein Molekül des Blutfarbstoffes aufzunehmen, untersuchte ich, ob etwa das reducirte Hämoglobin in der Lage sei, die doppelte Cyanmenge aus Cyangas festzuhalten.

Ursprünglich von der Vorstellung ausgehend, zwei hypothetische Hydroxylgruppen ${ }^{1}$ ) seien im Methämoglobin zum Austausche gegen Cyan bereit, hoffte ich direkt aus Hämoglobin die Verbindung $\mathrm{Hb}=(\mathrm{CN})_{2} \mathrm{zu}$ erhalten $(\mathrm{Hb}$ als Symbol des Hämoglobinkernes).

Zur Durchführung dieses Versuches wurde einerseits eine grössere Menge Oxyhämoglobin vom Pferde, in Wasser suspendirt, in einem dickwandigen, verschlossenen Kolben zur Aufzehrung des Sauerstoffes längere Zeit sich überlassen und nach der vollständigen Reduction evacuirt. Andererseits bereitete ich mir Cyangas aus scharf getrocknetem Quecksilbercyanid und bewahrte dieses in einem trockenen Gasometer über Quecksilber auf.

Beim Einleiten des Cyangases auf das Hämoglobin blieb dessen Lösung Anfangs unverändert; erst allmählich, etwa in einer Viertelstunde, begann die Umwandlung der Purpurfarbe in ein helles Roth und durch spectroskopische Beobachtung liess sich die Bildung von Cyanhämoglobin vermuthen. Wird der Ueberschuss des Cyangases, nachdem diese Farbumwandlung vor sich gegangen ist, durch Auspumpen entfernt, so blieb

1) Vergl. Du Bois' Arch. 1899. S. 486. 
die Blutfarbstofflösung auch bei nachfolgendem Zutritt von Luft klar; bei längerem Verweilen des Cyangases in der Lösung tritt jedoch bald eine Trübung derselben ein, rascher noch, wenn gleichzeitig Luft hinzutritt. Schliesslich setzte sich in der Lösung ein reichlicher Niederschlag ab. Ob derselbe durch eine dem Dicyan anhaftende Verunreinigung oder durch Zersetzung desselben entsteht, vermochte ich nicht zu entscheiden.

Die nach der Entfernung des überschüssig zugesetzten Cyangases klar gebliebene Flüssigkeit gab in einigen Fällen nach gutem Einkühlen und aut Zusatz von $1 / 4$ ihres Volumens starkem Weingeiste recht zerfliessliche, rothe Krystallnädelchen, welche mit der Centrifuge von der Mutterlauge getrennt, über Schwefelsäure und Natronkalk im luftverdünnten Raume getrocknet wurden.

Der Krystallwassergehalt der Präparate ergab sich in einem Falle zu etwa 5, in einem zweiten Falle zu etwa 7\%.

$4,6958 \mathrm{~g}$ der trockenen Krystalle gaben nach der früher beschriebenen Methode der Cyanbestimmung mittels Quecksilberoxyd

$0,0334 \mathrm{~g}$ Cyansilber $=0,0065 \mathrm{~g}$ Cyan oder 0,138 \% Cyan.

Die photometrische Untersuchung gab im Mittel aus drei Bestimmungen: $\mathrm{A}=0,001832, \mathrm{~A}^{\prime}=0,001516, \frac{\epsilon^{\prime}}{\epsilon}=\frac{\mathrm{A}}{\mathrm{A}^{\prime}}=1,209$, also die gleichen Werthe, wie sie für Cyanhämoglobin aus Ferricyankalium erhalten worden sind.

Es dürfte daher wohl keinem Anstande unterliegen, dieses Blutfarbstoffderivat als identisch mit dem Cyanhämoglobin genannten Körper zu erklären. Es sei noch erwähnt, dass diese Blutfarbstofflösungen nach dem Evacuiren deutlich nach Blausäure rochen.

Kohlenoxyd- und Stickoxydhämoglobinlösungen, über Quecksilber mit Cyangas gesättigt, zeigten keine Einwirkung, auch keine Aenderungen ihrer Spectra; Oxyhämoglobinlösungen gaben eine reichliche Gasentwicklung, behielten aber zwei Absorptionsstreifen im Spectrum und verwandelten sich erst nach folgendem Evacuiren in Cyanhämoglobin, welches auch krystal- 
lisirt aus diesen Lösungen erhalten werden konnte, während die gleiche Behandlung von Kohlenoxydhämoglobin - Sättigen mit Cyangas und nachheriges Auspumpen - kein einheitliches Produkt zu liefern schien.

Bei dem hohen Absorptionscoefficienten wässeriger Lösungen für Cyangas erscheint leider eine Messung der in Reaction tretenden Gasmenge so gut wie ausgeschlossen.

Da diese differenten Darstellungsweisen immer den gleichen Körper ergaben, da ferner in der Litteratur keine irgendwie verlässliche Angabe vorhanden ist, dass ein anderes als das oben beschriebene Cyanderivat des Blutfarbstoffs dargestellt ist, so glaube ich, dass der Name Cyanhämoglobin diesem zweckmässig zukommen kann.

Ehe wir mit Erfolg versuchen können, diese Anlagerung der Blausäure resp. des Cyans durch eine Formel darzustellen, muss erst die Vorfrage nach der Constitution des Methämoglobins gelöst sein, da die rasche Anlagerung der Blausäure an das letztere wieder einen wichtigen Unterschied zwischen Methämoglobin und Oxyhämoglobin bedeutet. Daher sind wir vorderhand nur in der Lage, die verschiedenen Möglichkeiten zu erörtern; viele können es wohl nicht sein nach den mannigfachen Details, welche wir über beide Körper kennen.

Vor etwa zwei Jahren ${ }^{3}$ ) wurde im Hinblick auf die Lostrennung des gesammten, molekular gebundenen Oxyhämoglobinsauerstoffs bei der mittels Ferricyankalium erfolgenden Methämoglobinbildung die Vermuthung ausgesprochen, dass die Atomgruppen, welche an die Stelle des Sauerstoffmoleküls eintreten, zwei, natürlich einander gleichwerthige Hydroxylgruppen sein mögen.

Ihre Stütze fand diese Ansicht in dem entschieden sauren Charakter des Methämoglobins, der sich augenfällig durch die Verschiedenheit des Methämoglobinspectrums in neutraler (resp. saurer) und alkalischer Flüssigkeit erweist. Was nun letzteren Unterschied bei saurer und bei alkalischer Reaction bewirkt, muss im Cyanhämoglobin verschwunden sein. Wären nun

1) a. a. 0 . S. 486 . 
zwei einander gleichwerthige Hydroxylgruppen im Methämoglobin vorhanden, so müssten an ihrer Stelle auch zwei Cyangruppen eintreten, bis der auffallende Säurecharakter verschwunden ist.

Auffallend ist die Festigkeit, mit welcher das Cyan im Cyanhämoglobin gebunden ist. Aus den Lösungen vermag weder das Vacuum noch anhaltendes Durchleiten indifferenter Gase, selbst bei gleichzeitigem Erwärmen auf $40^{\circ}$, das Cyan herauszureissen; auch auf die trockene Verbindung wirkt das Vacuum bis zur Temperatur von $40^{\circ}$ nicht ein.

Andererseits verleiht der Eintritt dieser Cyangruppe dem Blutfarbstoffmolekül selbst eine Resistenz, welche in mancher Hinsicht grösser $\mathrm{zu}$ sein scheint als jene, welche durch die Kohlenoxydgruppe im Kohlenoxydhämoglobin bewirkt ist. Man kann Cyanhämoglobinlösungen tagelang ohne Schutz vor dem Zutritt von Bakterien bei Zimmertemperatur der Luft aussetzen, ohne eine Veränderung wahrnehmen zu können. Erst nach wochenlangem Stehen solcher Lösungen, unter fortwährendem Ersatz des verdunsteten Wassers, konnte ich das Auftreten von Fäulniss und dann die Umwandlung in reducirtes Hämoglobin beobachten, welches, an der Luft geschüttelt, in Oxyhämoglobin überging. Besonders auffallend erschien mir, dass geringe Mengen von Hydrazinhydrat sich ganz unwirksam erwiesen, ebenso verdünnte Lösungen von Hydroxylaminsulfat, welche mit überschüssigem Natriumcarbonat versetzt waren. Ein beträchtlicher Zusatz von Hydrazinhydrat bewirkt eine tiefgehende.Zerstörung unter Bildung hellgrüner Flüssigkeiten, die nicht beim Hämochromogen halt macht. ${ }^{1}$ )

Eine glatte Rückverwandlung des Gyanhämoglobins in Hämoglobin ist durch Schwefelwasserstoffgas zu erreichen beim Einleiten dieses Gases in eine von absorbirtem Sauerstoff vollS. 498 .

1) Vergl. Hüfner, Arch. f. An. u. Physiol., physiol. Abth., 1900, 
kommen freie Lösung des Cyanhämoglobins. Will man aus dieser Flüssigkeit Oxyhämoglobin gewinnen, so muss aus ihr, ehe man Sauerstoff zuströmen lässt, auf das. Sorgsamste der Schwefelwasserstoff entfernt werden, in der gleichen Weise, wie bei der Rückverwandlung des Methämoglobins in $0 x y-$ hämoglobin.

Auf Zusatz von Mineralsäuren tritt rasch eine Braunfärbung der rothen Cyanhämoglobinlösung ein, welche auch auf weiteren Zusatz von Blausäure nicht verschwindet; aus concentrirten Lösungen fällt ein brauner Niederschlag, der in Laugen gelöst auf weiteren Zusatz von Hydrazinhydrat das typische Hämochromogenspectrum gibt.

Ein Versuch, nach der Schalfejew'schen Methode durch Erwärmen des Cyanhämoglobins mit cyanwasserstoffhaltigem Eisessig ohne Gegenwart von Chloriden eine krystallisirte, dem Hämin entsprechende Verbindung zu erhalten, gab keine Krystalle, während auf Zusatz von Chlornatrium nach Schalfejew in reichlicher Ausbeute Häminkrystalle erhalten wurden.

Auf Grund der Bemerkung, die Hoppe-Seyler in seinem Lehrbuch der physiologischen Chemie, Seite 384, macht, dass nämlich Wasserstoffsuperoxyd das 0xyhämoglobin unverändert lässt, die Cyanwasserstoffverbindung desselben in Cyanhämatin und Eiweiss zerlegt, habe ich die Reaction des Cyanhämoglobins mit Wasserstoffsuperoxyd sorgfältig beobachtet. Hoppe's Bemerkung schien darauf hinzuweisen, dass aus dem Cyan resp. der Blausäure durch Oxydation eine auf Blutfarbstoff zersetzend wirkende Säure entstünde. Ich habe reine Lösungen von Cyanhämoglobin, welche keine überschüssige Blausäure enthielten, mit Wasserstoffsuperoxydlösungen zusammengebracht, welchen soviel Soda zugefügt war, dass letztere Lösungen schwach alkalisch waren. Beim Zusammenbringen der beiden Flüssigkeiten konnte ich niemals eine Hämatinabspaltung beobachten, doch trat, allerdings bei grösserem Zusatz von Wasserstoffsuperoxyd, eine eingreifende Veränderung ein; es entfärbte sich nämlich die Blutfarbstofflösung ganz allmählich und schliesslich wurde eine hellgrüne Flüssigkeit erhalten. Die gleiche Veränderung ist bei der Wirkung von Wasserstoff-

Hoppe-Seyler's Zeitschrift f. physiol. Chemie. XXXIII. 
superoxyd auf Oxyhämoglobin und Methämoglobin zu. beobachten. ${ }^{1}$ )

Die Giftwirkung des Cyanhämoglobins ist jedenfalls eine geringe. Herr Dr. Rudolf Freiherr von Seiller hatte die Freundlichkeit, $6 \mathrm{~g}$ desselben einem $8 \mathrm{~kg}$ schweren Hunde einzugeben. Derselbe blieb auf diese Dosis vollkommen munter.

Als die wesentlichen Resultate dieser Arbeit ergeben sich folgende Sätze:

1. Es existirt ein krystallisirtes, gut krystallisirbares Cyanhämoglobin, welches in einem Molekül ein Molekül Blausäure oder das einwerthige Cyanradical fest gebunden enthält.

2. Diese Cyangruppe kann nur (mittelbar oder unmittelbar) eine jener Valenzen sättigen, an denen im Oxyhämoglobin das locker gebundene Sauerstoffmolekül haftet.

3. Das reine Methämoglobin ist lichtbeständig.

4. Bock's Photomethämoglobin ist Cyanhämoglobin, es entsteht durch die Wirkung der Blausäure, welche aus dem Ferricyankalium durch das Sonnenlicht abgespalten wird.

Wien, im August 1901.

1) Auch Hämatin wird durch reichlichen Zusatz von Wasserstoffsuperoxyd entfärbt, ohne dass durch das Spectroskop, mit Ausnahme der Aufhellung, eine Aenderung des Spectrums bemerkbar wäre. Die gleiche Einwirkung zeigt sich bei der Electrooxydation, über welche gelegentlich berichtet werden soll. 